\title{
Stoichiometric Fingerprinting as an Aid in Understanding Complex Reactions: The Oxidation of Malonic Acid by Cerium(IV)
}

\author{
Bettina Neumann,,$+\star$ Oliver Steinbock,,,$+ \neq$ Stefan C. Miiller,,$\$$ and Nar S. Dalal ${ }^{\dagger}$ \\ Department of Chemistry, Florida State University, Tallahassee, Florida 32306-3006, \\ Institut für Experimentelle Physik, Otto-von-Guericke-Universität Magdeburg, Universitätsplatz, 2, \\ D-39106 Magdeburg, Germany, and Max-Planck-Institut für molekulare Physiologie, \\ Rheinlanddamm 201, D-44139 Dortmund, Germany
}

Received: December 6, 1996; In Final Form: February 19, $1997^{\otimes}$

\begin{abstract}
The stoichiometry of the Ce(IV) oxidation of malonic acid (MA) under aerobic and anaerobic conditions was investigated. The $\mathrm{Ce}(\mathrm{IV}) / \mathrm{MA}$ consumption ratio is found to vary from about 3.5 to 7 , depending nonlinearly on the initial concentration of malonic acid as well as dissolved oxygen. The observed data can be quantitatively explained by a model that employs the characteristic structure of branching pathways in the overall mechanism and does not require the exact knowledge of the rate constants. It is concluded that a systematic study of consumption ratios provides an important aid for elucidating mechanisms of complex reactions.
\end{abstract}

The study of stoichiometries and reaction mechanisms of the oxidation of organic acids first gained considerable interest during the development of analytical methodologies such as redox titrations. ${ }^{1}$ In this respect the oxidation of malonic acid (MA) by ceric (Ce(IV)) ions has become a textbook example, where the overall reaction is written as

$$
\begin{aligned}
& \mathrm{CH}_{2}(\mathrm{COOH})_{2}+ 6 \mathrm{Ce}(\mathrm{IV})+2 \mathrm{H}_{2} \mathrm{O} \rightarrow \\
& \mathrm{HCOOH}+6 \mathrm{Ce}(\mathrm{III})+2 \mathrm{CO}_{2}+6 \mathrm{H}^{+}
\end{aligned}
$$

However, recent product analyses under anaerobic conditions revealed significant deviations from the above value of 6 for the $\mathrm{Ce}(\mathrm{IV}) / \mathrm{HCOOH}$ ratio, ${ }^{3}$ and also older literature values for the stoichiometric coefficient, $\mathrm{Ce}(\mathrm{IV}) / \mathrm{MA}$, are known to scatter in the range of 5.9-6.6. ${ }^{4-6}$ For a similar reaction, the oxidation of methylmalonic acid by $\mathrm{Ce}(\mathrm{IV})$, the reported value of 4.7 represents an even larger deviation with respect to the predicted value of $6 .^{7}$ A first clue toward the understanding of some of these discrepancies was found in the case of the oxidation of malonic acid by manganic pyrophosphate. Here a change in stoichiometry due to the presence of molecular oxygen was measured. ${ }^{8}$ Barkin et al. ${ }^{5}$ were the first to notice an oxygen influence on reaction $\mathrm{R} 1$ during their kinetic studies, and more recently Neumann et al. were able to identify an oxygen-specific intermediate. ${ }^{9}$ All these points put the general validity of R1 into question.

Since stoichiometry is one of the most important attributes of a reaction, we undertook a systematic, quantitative investigation with the goal of obtaining clues to the cause of this "variable stoichiometry" issue and its relation to dissolved oxygen. This Letter summarizes our results for the Ce(IV) oxidation of MA, the above reaction $\mathrm{R} 1$. $\mathrm{Ce}(\mathrm{IV}) / \mathrm{MA}$ was selected as the test case because (i) there are earlier, quantitative data available on its stoichiometry, but which need mechanistic details, and (ii) it is the organic subset of the Belousov-Zhabotinsky reaction, ${ }^{10}$ which is the subject of current research activity, because it is one of the most prominent model systems for nonlinear phenomena, including oscillations and spatiotemporal self-

\footnotetext{
$\dagger$ Florida State University.

$¥$ Otto-von-Guericke-Universität Magdeburg.

$\S$ Max-Planck-Institut für molekulare Physilogie.

${ }^{\otimes}$ Abstract published in Advance ACS Abstracts, April 1, 1997.
}

organization. ${ }^{11}$ Our experimental data reveal an unexpectedly large and nonlinear dependence of the consumption ratio on the initial malonic acid concentration as well as oxygen content. Comparison of these data with numerical simulations provides strong indications that the measured function of consumption ratios is, indeed, a stoichiometric fingerprint, which should be of general importance as an aid in distinguishing between various pathways of complex reactions.

Spectrophotometric as well as titration methods were applied for the determination of the ratios between the consumed amounts of $\mathrm{Ce}(\mathrm{IV})$ (used as $\mathrm{Ce}(\mathrm{IV})\left(\mathrm{SO}_{4}\right)_{2} \times 4 \mathrm{H}_{2} \mathrm{O}$, Merck) and the initial malonic acid concentration (doubly recrystallized from acetone). All measurements were performed in $1 \mathrm{M} \mathrm{H}_{2} \mathrm{SO}_{4}$ at room temperature. The absorbance of residual $\mathrm{Ce}$ (IV) was measured photometrically using a Hewlett-Packard HP 8453 UV/vis diode array spectrophotometer. Titration of the residual $\mathrm{Ce}$ (IV) concentrations were carried out using standard $\mathrm{Fe}(\mathrm{II})$ $\left(\mathrm{NH}_{4}\right)_{2}\left(\mathrm{SO}_{4}\right)_{2}$ (Fischer), with ferroin (Fluka) as indicator. For the experiments under nitrogen atmosphere, the stock solutions were preequilibrated with nitrogen and transferred into an nitrogen-filled glovebox to start the reactions. For the experiments under aerobic conditions, the stock solutions were preequilibrated with air. Comparison to experiments without preequilibration, however, gave no significantly different results. The samples were analyzed after at least 3 days up to 1 week after the start of the reaction. The initial malonic acid concentrations were varied between 0.01 and $10 \mathrm{mM}$ with initial $\mathrm{Ce}(\mathrm{IV})$ concentrations between 0.5 and $75 \mathrm{mM}$.

Figure 1 summarizes the data obtained. Here the determined consumption ratio $f$, which is defined as

$$
f \equiv[\mathrm{Ce}(\mathrm{IV})]_{\text {consumed }}:[\mathrm{MA}]_{\text {initial }}
$$

is plotted against the initial malonic acid concentration of each experiment. The circles in Figure 1 represent experiments analyzed by redox titration, whereas the triangles correspond to results obtained from photometry, both under aerobic conditions. Photometric and titration results are in good agreement. The striking feature of these data is that the ratio $f$ is not a fixed value but varies in a nonlinear fashion. Starting with a slow increase of $f$ from about 3 to 4 at very low concentrations of malonic acid, $f$ changes with a relatively distinct turning point at $\log \left([\mathrm{MA}]_{\text {initial }} / \mathrm{mM}\right) \approx-0.4$ along a 


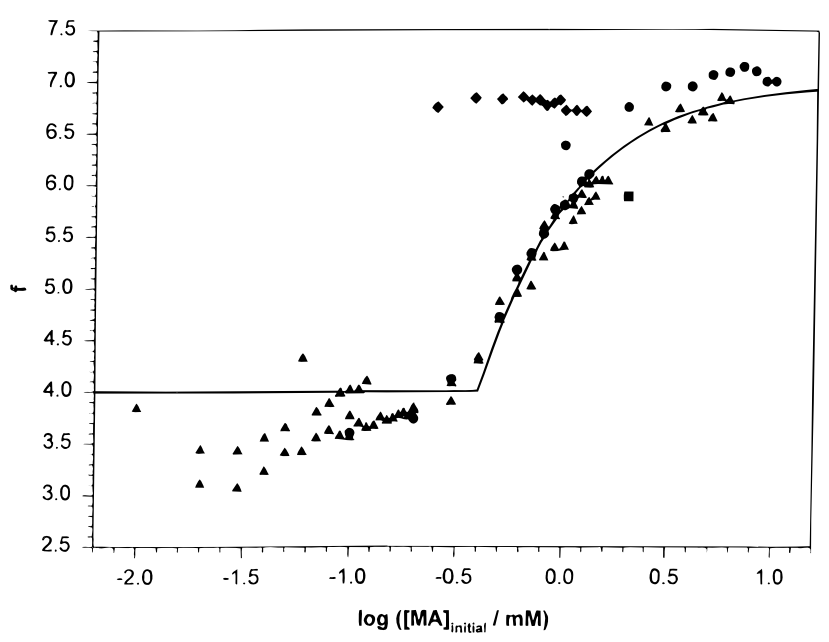

Figure 1. Dependence of the consumption ratio $f$ on the initial malonic acid concentration and the atmospheric conditions in semilogarithmic presentation. Experimental data and the superimposed result of the model shown as symbols (aerobic conditions, $\boldsymbol{\Delta}$ photometry, $\bullet$ titration; anaerobic conditions, $\bullet$ from ref 4) and solid line, respectively.

concave curve to a final value of about 7 . For the malonic acid concentration range with the most pronounced change in $f$, we also performed measurements under nitrogen atmosphere (diamonds in Figure 1). Within the experimental error their consumption ratios are about 7 , with essentially no dependence on the malonic acid concentration. For comparison of our data to previous measurements we also include the value of 5.9 (square in Figure 1) reported by Jwo and Noyes, ${ }^{4}$ which can be seen to fall into a reasonable interval of the present data under aerobic conditions.

The presented results reveal a complex dependence of the overall stoichiometry on the initial malonic acid and oxygen concentration. To explain this behavior we assume that our experimental data exhibit a typical pattern occurring as a result of competing reaction pathways with different probabilities. These probabilities can depend on the concentration of the involved substrates and/or intermediates, the rate of their reaction, and the stoichiometry of their reaction products. The key process of our previously proposed mechanism of the aerobic oxidation of malonic acid by ceric ions is the competition between an anaerobic and an aerobic pathway leading to the formation of 1,1,2,2-ethanetetracarbonic acid (ETA) and the peroxymalonyl radical $\left(\mathrm{MAOO}^{\circ}\right)$, respectively. ${ }^{9}$ This feature is summarized by the following simple reaction mechanism

$$
\begin{aligned}
& \mathrm{Ce}(\mathrm{IV})+\mathrm{MA} \rightarrow \mathrm{Ce}(\mathrm{III})+\mathrm{MA}^{\bullet}+\mathrm{H}^{+} ; \\
& k_{2}=0.3 \mathrm{M}^{-1} \mathrm{~s}^{-1} \\
& \mathrm{MA}^{\bullet}+\mathrm{MA}^{\bullet} \rightarrow \mathrm{ETA} ; k_{3}=4.2 \times 10^{8} \mathrm{M}^{-1} \mathrm{~s}^{-1} \\
& \mathrm{MA}^{\bullet}+\mathrm{O}_{2} \rightarrow \mathrm{MAOO}^{\bullet} ; k_{4}=1.7 \times 10^{7} \mathrm{M}^{-1} \mathrm{~s}^{-1} \\
& \mathrm{MAOO}^{\bullet}+\mathrm{MAOO}^{\bullet} \rightarrow \mathrm{TA}+\mathrm{GOA}+\mathrm{CO}_{2}+\mathrm{O}_{2} ; \\
& k_{5}=2.2 \times 10^{8} \mathrm{M}^{-1} \mathrm{~s}^{-1}
\end{aligned}
$$

where $\mathrm{MA}^{\bullet}$, TA, and GOA are the malonyl radical, tartronic acid, and glyoxylic acid, respectively. A schematic presentation of this mechanism is shown in Figure 2. Our focus lies on the first branching point of the mechanism, with the malonyl radical as the key species. The major pathways exhibit different consumption ratios $f_{i}$. This can lead to alterations of the overall reaction stoichiometry depending on the fraction of the malonyl radicals forming $\mathrm{TA}$ and GOA after reacting with oxygen

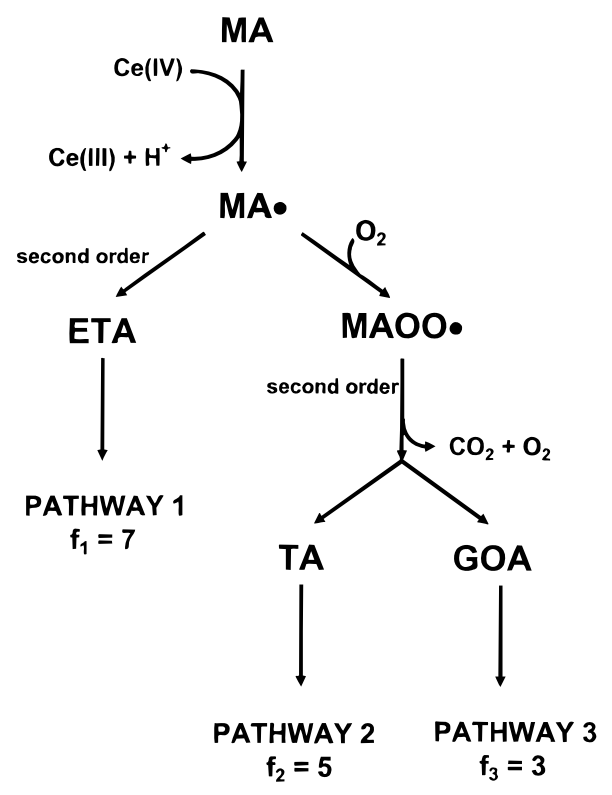

Figure 2. Schematic drawing of the simple reaction model used for numerical calculation (including the stoichiometries $f$ of each pathway).

compared to the amount of ETA produced by dimerization of the malonyl radical. To model this initial part of the unknown complete mechanism, we further have to take into account the stoichiometries of the ceric oxidations of the intermediates (ETA, TA, GOA). Preliminary experiments revealed a stoichiometry of 12 for the Ce(IV) oxidation of ETA. In the cases of TA und GOA, we used the literature values ${ }^{4,12}$ of 4 and 2, respectively. The rate constants were chosen as reported previously (see also R2-R5). ${ }^{9}$ In order to evaluate the consumption ratio as a function of initial conditions, we first relate $f$ to the probabilities $p_{i}(i=1,2,3)$ describing the likelihood for a malonic acid molecule to be oxidized along the $i$ th pathway

$$
f=[\mathrm{Ce}(\mathrm{IV})]_{\text {consumed }} /[\mathrm{MA}]_{\text {initial }}=7 p_{1}+5 p_{2}+3 p_{3}
$$

where the integer weights correspond to the pathway-characteristic stoichiometries $f_{i}$. For pathway 1 (oxidation via ETA) the weight $f_{l}=7$ reflects the fact that 2 malonic acid molecules and 2 ceric ions are necessary for the formation of 1 ETA molecule, which is then oxidized by 12 additional ceric ions. Hence, the resulting value is $f_{l}=[\mathrm{Ce}(\mathrm{IV})]_{\text {consumed }} /[\mathrm{MA}]_{\text {initial }}$ via ETA $=(2+12) / 2=7$. The weight factors for pathways 2 and 3 are calculated accordingly. Furthermore, the total sum of probabilities has to be $1\left(\sum_{i=1}^{3} p_{i}=1\right)$ and $p_{2}=p_{3}$, since equal amounts of TA and GOA are produced. In the framework of the mechanism $\mathrm{R} 2-\mathrm{R} 5$, we can reconstruct the probabilities $p_{i}$ from the final product concentrations as

$$
\begin{aligned}
p_{1}=2[\mathrm{ETA}]_{\infty} /[\mathrm{MA}]_{\text {initial }} ; \quad p_{2}=[\mathrm{TA}]_{\infty} /[\mathrm{MA}]_{\text {initial }} ; \\
p_{3}=[\mathrm{GOA}]_{\infty} /[\mathrm{MA}]_{\text {initial }}
\end{aligned}
$$

The task of evaluating $f$ is now reduced to the calculation of $[\mathrm{ETA}]_{\infty},[\mathrm{TA}]_{\infty}$, and $[\mathrm{GOA}]_{\infty}$. These values were obtained by numerical integration of the rate equations for the reactions R2R5 using the facsimile program. The numerical result is presented as the solid line in Figure 1. It can be seen to be in good agreement with the main features of the experimental data under aerobic conditions. To check the sensitivity of the model against changes in the atmospheric conditions, we calculated the consumption ratios as described above for different initial oxygen concentrations. The numerical results, shown in Figure 3 , reveal a continuously shifting turning point as a consequence 


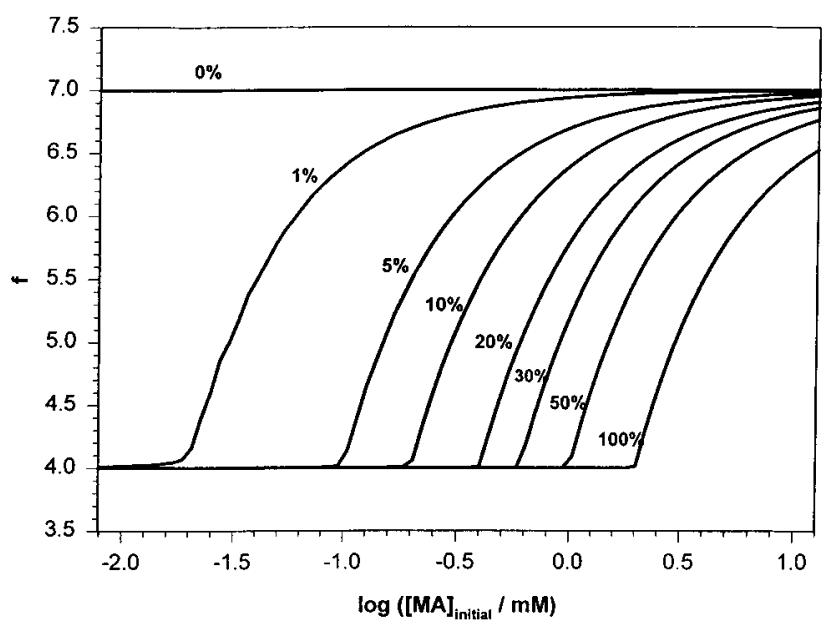

Figure 3. Dependence of the consumption ratio $f$ on the initial malonic acid and oxygen concentrations. 100\% oxygen equilibration is estimated to be an effective oxygen concentration of $1 \mathrm{mM}$. Results of the model calculations are based on the reactions R2-R5 (see text).

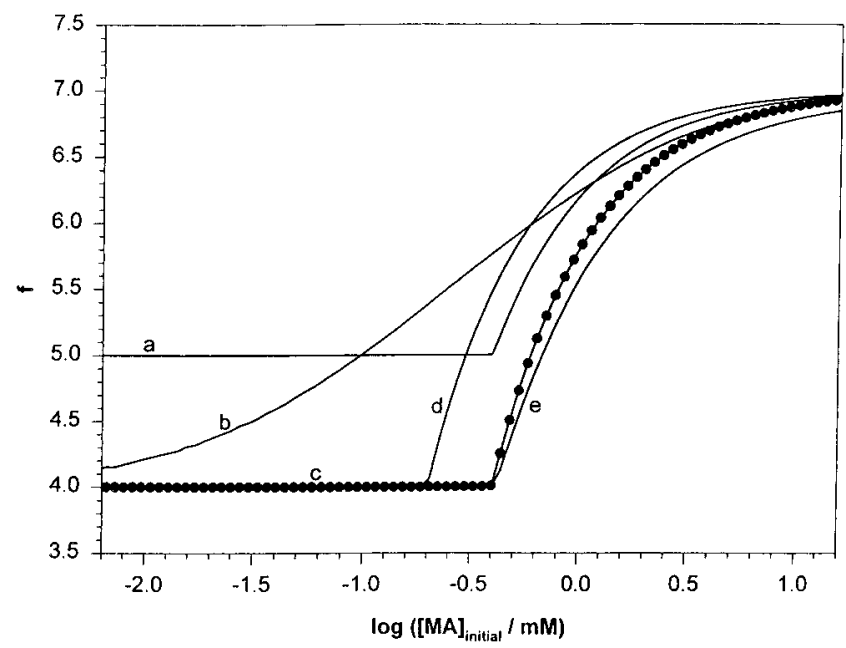

Figure 4. Numerical results obtained by modifying the original model or the initial conditions. The solid circles represents the original model (cf. Figure 1). (a) Exchange of glyoxylic acid to mesoxalic acid; (b) and (c) rate constants of the formation of the peroxymalonyl radical set to $1.7 \times 10^{5}$ and $1.7 \times 10^{9} \mathrm{M}^{-1} \mathrm{~s}^{-1}$, respectively; (d) no oxygen release during the decay of the peroxymalonyl radical; (e) implementation of an additional decay pathway for the malonyl radical.

of the change in initial oxygen concentrations. The initial consumption ratio of 4 is governed by the aerobic pathway, whereas the straight line at $f=7$ represents the anaerobic pathway. The turning points in the curves of different initial oxygen concentrations define the condition at which oxygen is consumed completely. Furthermore, we performed extensive simulations to study the sensitivity of the model to variations in rate constants, changes in product formation, and additional pathways. The main results of these studies are summarized in Figure 4. The model turns out to be quite robust against changes in rate constants, usually over several orders of magnitude. The solid circles describe the original model and rate constants (R2-R5) (cf. Figure 2). We modified, for example, the rate constant $k_{4}$ for the formation of the peroxymalonyl radical, up to $1.7 \times 10^{9} \mathrm{M}^{-1} \mathrm{~s}^{-1}$ (curve c, Figure 4), which introduces practically no change, and down to $1.7 \times 10^{5}$ $\mathrm{M}^{-1} \mathrm{~s}^{-1}$ (curve b), where we observed the first significant smoothing of the plot, due to the competition of this reaction with the formation of ETA. For curve a (Figure 4) we replaced glyoxylic acid by mesoxalic acid. The stoichiometry of mesoxalic acid 4 is known to be 4 , which means a higher overall stoichiometry of the aerobic pathway. Consequently, we see that the plot starts with the expected value of 5. For curve d, the production of oxygen during the decay of the peroxymalonyl radical was excluded. It can be seen that the turning point shifts significantly to lower values of $[\mathrm{MA}]_{\text {initial }}$, accounting for the decreased availability of oxygen (cf. Figure 3 ). This finding strongly supports the proposed oxygen release during the peroxy radical decay. Finally, we included an additional decay mechanism for the malonyl radical: the direct decarboxylation of the malonyl radicals into acetic acid radicals forming succinic acid by recombination. ${ }^{13}$ We found that this additional pathway, however, introduces only small changes and would be of minor importance for the realized stoichiometries (curve e).

This report documents the following: For the Ce(IV) oxidation of MA, the earlier reported range of consumption ratios (5.9-6.6) around the textbook value of 6 is not primarily caused by experimental errors but can be rather understood as the result of a competition between anaerobic and aerobic reaction pathways. The complete range of consumption ratios is found to be between approximately 3.5 and 7 with a distinct dependence on the initial concentrations of malonic acid and oxygen, which can be quantitatively modeled on the basis of only four main reactions. In a broader context, this study opens a new avenue for the analysis of complex reaction mechanisms. In particular, reactions with high stoichiometries have the potential to show a significant dependence of consumption ratios on initial parameters. The systematic measurement of this dependence should allow, analogous to the presented case, the extraction of the important information on branching reaction pathways, which can be considered to be a fingerprint of the mechanism.

Acknowledgment. This research was supported by the Deutsche Forschungsgemeinschaft and Florida State University. O.S. thanks the Fonds der Chemischen Industrie for a Liebig Fellowship.

\section{References and Notes}

(1) Richardson, W. H. In Organic Chemistry; Blomquist, A. T., Ed.; Academic Press: New York and London, 1965; Vol. 5 (Wiberg, K. B., Ed.), Part A, Chapter IV, pp 244.

(2) Harris, D. C. In Quantitative Chemical Analysis, 4th ed.; Freeman, W. H. and Company: New York, 1995.

(3) Gao, Y.; Försterling, H.-D.; Noszticzius, Z.; Meyer, B. J. Phys. Chem. 1994, 98, 8377. Gao et al. performed HPLC experiments with a $6: 1$ initial concentration ratio of Ce(IV) and MA. Only $27 \%$ of the expected formic acid concentration was found.

(4) Jwo, J.-J.; Noyes, R. M. J. Am. Chem. Soc. 1975, 97, 5422.

(5) Barkin, S.; Bixon, M.; Noyes, R. M.; Bar Eli, K. Int. J. Chem. Kinet. 1978, 10, 619

(6) Willard, H. H.; Young, P. J. Am. Chem. Soc. 1930, 52, 132.

(7) Ruoff, P.; Nevdal, G. J. Phys. Chem. 1989, 93, 7802-7806.

(8) Drummond, A. Y.; Waters, W. A. J. Chem. Soc. 1954, 2456.

(9) Neumann, B.; Müller, S. C.; Hauser, M. J. B.; Steinbock, O.; Simoyi, R. H.; Dalal, N. S. J. Am. Chem. Soc. 1995, 117, 6372.

(10) Field, R. J.; Burger, M., Eds. Oscillations and Traveling Waves in Chemical Systems; Wiley-Interscience: New York, 1985.

(11) Swinney, H. L., Krinsky, V. I., Eds. Waves and patterns in chemical and biological media; Elsevier: Amsterdam, 1991 (conference issue: Physica D 1991, 49).

(12) Neumann, B.; Steinbock, O.; Müller, S. C.; Dalal, N. S. J. Phys. Chem. 1996, 100, 12342.

(13) Försterling, H.-D.; Pachl, R.; Schreiber, H. Z. Naturforsch. 1987, $42 a, 963$. 